\title{
Ibuprofen treatment for patent ductus arteriosus in preterm infants: a retrospective cohort study in a leading Chinese center
}

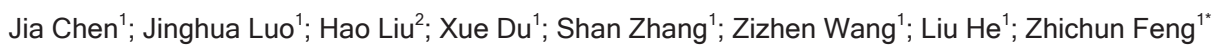

\begin{abstract}
Objective There is a dilemma of ibuprofen treatment with patent ductus arteriosus (PDA) as to how and when to treat. We aimed to clarify this issue in very preterm infants (VPIs; $<32$ weeks). Methods: This retrospective study included 1659 VPIs who were diagnosed with PDA according to echocardiographic examinations and cardiovascular dysfunction scoring system (the CVD scoring). The VPIs were classified into six groups (A1, A2, A3, B1, B2, and B3) based on CVD scores (A, < 3 , and $\mathrm{B}, \geq 3$ ), and treatment with ibuprofen for PDA (1, conservational management; 2 , early ibuprofen treatment; and 3 , late ibuprofen treatment). Treatment was stopped when PDA was closed, CVD score was zero or PDA needed ligation. Results: VPIs with CVD scores < 3 had most PDA closure without surgery, and early ibuprofen treatment did not significantly affect PDA closure. VPIs with CVD scores $\geq 3$ had some PDA closure after 2 courses of treatment, but closure rates decreased linearly with ibuprofen course $\left(1^{\text {st }} 75.2 \%, 2^{\text {nd }} 62.3 \%, 3^{\text {rd }} 50.0 \%, P<\right.$ 0.0001 ), and early ibuprofen treatment (group B2) did not increase PDA closure compared to late ibuprofen treatment (group B3). In these same infants, the longer they were in CVD scores $\geq 3$, the more the complications of preterm were increased (retinopathy of prematurity ROP $1^{\text {st }} 16.5 \%, 2^{\text {nd }} 23.8 \%, 3^{\text {rd }} 29.6 \%, P=0.016$; bronchopulmonary dysplasia BPD $1^{\text {st }} 15.5 \%, 2^{\text {nd }} 26.7 \%, 3^{\text {rd }} 33.8 \%, P<0.0001$; intraventricular hemorrhage IVH $\left.1^{\text {st }} 20.4 \%, 2^{\text {nd }} 32.4 \%, 3^{\text {rd }} 23.8 \%, P=0.015\right)$. Conclusion: lbuprofen is suggested for PDA closure when the PDA reopens or has developed into the stage when the CVD score $\geq 3$.
\end{abstract}

Keywords

preterm infants; ibuprofen; patent ductus arteriosus; cohort study; CVD score

Received 14 April 2021 accepted 20 July 2021

\section{What is already known}

The presence of PDA is often associated with various morbidity in small preterm infant. However, the assessment of PDA shunt often requires serial echocardiogram. The pediatrician and neonatologist in practice for whom these measurements are not constantly available must rely on clinical evaluation for management. This is a very common practice in underdeveloped and developing countries.

\author{
1 Department of Neonates, Senior Department of Pediatrics, the Seventh Medical \\ Center of PLA General Hospital, Beijing 100027, China \\ ${ }^{2}$ Nanfang Hospital, Southern Medical University, Guangzhou 510515, China \\ *Corresponding author Prof. Zhichun Feng, E-mail: zhjfengzc@126.com
}

\section{What this study adds}

The CVD scoring is a simple, easy-to-assess clinical features that can be used by family practitioner, general pediatrician, nursing practitioner and medical personnel for taking care critical patients. This work demonstrated that infant with a low CVD score (the cardiovascular distress of PDA < 3) had better outcomes, in terms of BPD, IVH and ROP. We believe that these important findings can be used as an adjunct in the evaluation and management of PDA when constant echocardiograms are not available. 


\section{Objective}

The patency of ductus arteriosus is essential for maintaining fetal circulation. The ductus arteriosus usually closes between 18 and 24 hours after birth in full-term infants but not in preterm neonates[1-2]. The presence of left to right shunting via the ductus may cause hemodynamic changes and respiratory distress[1, 3-4], which may affect the immediate and long term outcomes[5]. The continuous changes of the existing underlying lung pathology of respiratory distress syndrome (RDS) along with the ductus hemodynamics will make the clinical condition further complicated[6-8]. Therefore, in preterm infants with sustained patent ductus arteriosus (PDA), strategies for closing the ductus are needed. One such strategy is administration of ibuprofen. However, the optimal intervention time with ibuprofen remains yet to be determined for very preterm infants (VPI)[1, 9-11]. Therefore, we conducted the leading center study of VPIs to evaluate the PDA outcomes in VPIs, using the Cardiovascular Dysfunction Scoring System (CVD score)[12] as a tool to help determine the optimal regimen and intervention time for ibuprofen in a Chinese cohort of 2617 VPIs.

\section{Methods}

\section{Study design}

This retrospective cohort study included 2617 consecutive VPIs admitted into the Neonatal Intensive Care Unit (NICU) of the Seventh Medical Center of PLA General Hospital between January 1, 2015 and December 31, 2018.

VPIs were assessed for severity of hemodynamic disorders using a scoring system (the CVD scoring) as previously described by Yeh et al[13]. The total score was obtained by adding the separate scores for each of the 5 criteria: heart rate $(0,<160 /$ min; 1, 160 - 180/min; and 2, > 180/min); duration of murmur (0, normal; 1 , systolic; and 2, murmur continuing to diastole); quality of peripheral arterial pulsation ( 0 , normal; 1 , bounding brachial; and 2, bounding brachial and dorsalis pedis); and degree of precordial pulsation ( 0 , none; 1 , palpable; and 2, visible). These criteria were assessed and recorded in our NICU sheet as routine practice. CVD score has been shown to be well correlated with ductal shunt as demonstrated by echocardiogram(14)(citations and references). Infant with a CVD score $\geq 3,91 \%$ of infant was associated with left atrium/aortic root (LA/Ao) ratio $\geq 1.3$ and left ventricle end diastolic diameter (LVEDD) $\geq 1.35 \mathrm{~cm} / \mathrm{Kg}$, indicating a significant ductal shunt[14].

\section{Inclusion and exclusion criteria}

The inclusion criteria for the present study were: (1) infant with gestational age of < 32-weeks infants; (2) with diagnosed ultrasonically with PDA, regardless of the presence of hemodynamic changes. The exclusion criteria were (1) congenital anomalies, including other associated congenital heart diseases; (2) lethal cardiopulmonary status or infection at time of PDA diagnosis.

\section{Diagnostic criteria and definitions for variables}

PDA diagnosis criteria included [2, 15] echocardiography diagnostic standards[15], and conclusive presence of PDA was ensured by bedside ultrasonic examination (Acclarix LX8) during the first 3 days of postnatal life. The result of ultrasonic examination was confirmed by an ultrasound doctor and a pediatric cardiologist.

CVD score [12] of the VPIs included in the present study was determined according to their symptoms and divided into two categories ( $\geq 3$ and $<3$ ) to reflect the extent of significant ductal shunt. Briefly, CVD score $<3$ indicates an obvious presence of PDA and a likelihood of murmur, precordial pulsation, or full pulse, and a CVD score $\geq 3$ indicates symptomatic PDA, which might have poor clinical significance. Related symptoms of PDA include sustained dependence on the ventilator, worsening respiration, increased recurrent apnea, and hypotension resulting from pulmonary hemorrhage, with pulmonary hemorrhage being the most specific clinical manifestation.

The diagnosis criteria for bronchopulmonary dysplasia (BPD)[16], intraventricular hemorrhage (IVH)[17], necrotizing enterocolitis (NEC)[18] and retinopathy of prematurity (ROP)[19] were consistent with previous reports.

\section{Grouping}

Based on CVD scores, the infants were divided into two groups[12]: Group-A: with a CVD score < 3; and Group-B: with a CVD score $\geq 3$. The infants in each group were further divided into 3 subgroups[2] based on, conservative management only, early ibuprofen therapy given within 24 hours after failure to conservative management, and late ibuprofen therapy(giving at $\geq$ 24 hours after failure to conservative management). As such, the participants were classified into six groups: A1, A2, A3, B1, B2, and B3.

\section{Medical treatment}

All infants were managed following a protocol currently used in our NICU. The protocol essentially followed the recommendation from European Consensus with mild modification[20]. The protocol focused essentially on respiratory care guideline, fluid and 
nutritional intake, indication for antibiotics administration, time for brain ultrasonography and eye ground examination. All infants with respiratory distress were given a trial of CPAP with appropriate $\mathrm{FIO} 2$ and pressure to maintain $\mathrm{O} 2$ saturation at $90-95 \%$. The infant was intubated, and surfactant was administered if the infant failed to CPAP or required FIO2>0.6 or with severe retraction. Respiratory set up was adjusted appropriately to maintain $\mathrm{O} 2$ saturation between 90 95\%. Once the infant could tolerate FIO2 $\geq 0.3$ through SIMV, weaning process was speeded up. All infant with PDA received a 24 hours of conservative management, including a restriction fluid intake to $80-100 \mathrm{ml} / \mathrm{kg} /$ day, maintenance of hematocrit values $\geq 35 \%$, and adjustment of ventilator set-up to maintain appropriate blood gases and acid-base balance, and maintenance of normal electrolytes and of serum Ca $\geq 7.5 \mathrm{mg} / \mathrm{dl}[14]$. In spite of the conservative management, if the infant continuously had the ductal murmur, oral administration of ibuprofen was started (10 mg/kg initially, then $5 \mathrm{mg} / \mathrm{kg}$ every 24 hours for a maximum of 3 courses or 9 doses). The loading dose for ibuprofen administration was $10 \mathrm{mg} / \mathrm{kg}$, followed by two doses of $5 \mathrm{mg} / \mathrm{kg}$ administered every 24 hours as a course. Since previous studies showed that oral ibuprofen may confer equal or improved efficacy of PDA closure compared to intravenous administration[21], ibuprofen was administered orally in this study.

\section{Primary outcomes}

The primary outcomes were two-fold: to determine the optimal timing of ibuprofen use and the incidence of PDA closure in those with early vs late ibuprofen use, and to assess the use of CVD score in predicting the value of patient selection and the incidence of PDA closure after ibuprofen use in those with high vs low CVD score.

\section{Secondary outcomes}

The secondary outcomes were incidences of preterm complications, including BPD, ROP, IVH, NEC and death.

\section{Statistical analysis}

Continuous variables were compared with t-test and chi-square test, and likelihood ratio or Fisher's exact tests were used for categorical variables. For relevance analysis, Mantel-Haenszel statistics were applied. Data were analyzed with SPSS20.0, and $P<0.05$ was considered statistically significant.

\section{Results}

\section{Incidence of PDA}

Among the 2617 VPIs, 1659 were diagnosed with PDA (63.4\%).
The incidence of PDA decreased with increased GA and birth weight.

\section{Spontaneous closure of PDA}

The incidences of spontaneous PDA closure for VPIs with CVD scores < $3(n=1019)$ were $73.1 \%(559 / 765)$. However, the patients whose CVD scores were $\geq 3(n=640)$ had $6.7 \%(2 / 30)$ incidence rate of spontaneous closure of PDA of (Figure 1).

\section{Ibuprofen treatment}

Among VPIs whose CVD scores were $<3$, the rates of PDA closure were $86.1 \%, 91.3 \%$, and $73.9 \%$ after $1^{\text {st }}, 2^{\text {nd }}$ and $3^{\text {rd }}$ course of ibuprofen, respectively. Among patients whose CVD scores were $\geq$ 3 , the rates of PDA closure were $75.2 \%, 62.3 \%$ and $50 \%$ after $1^{\text {st }}$, $2^{\text {nd }}$ and $3^{\text {rd }}$ course of ibuprofen, respectively (Table 1).

\section{Outcomes between patients with CVD score $<3$ and $\geq 3$}

Clinical Outcomes: The time to PDA closure among infants in the group with CVD scores $<3$ (group A) was shorter compared to that with CVD score (group B) $\geq 3(10.8 \pm 0.8 \mathrm{~d}$ vs $14.5 \pm 1.4 \mathrm{~d}, P$ $<0.0001$ ), and the DA diameter in infants with CVD scores $<3$ was smaller compared to that in those with CVD scores $\geq 3(2.2$ $\pm 0.5 \mathrm{~mm}$ vs $2.4 \pm 0.6 \mathrm{~mm}, P<0.0001)$. Moreover, the incidences of RDS $(66.8 \%$ vs $83.5 \%, P<0.0001)$, BPD $(8.9 \%$ vs $22.4 \%, P$ $<0.0001)$, ROP $(7.7 \%$ vs $20.7 \%, P<0.0001)$, and IVH $(17.4 \%$ vs $25 \%, P<0.0001)$ were lower in infants with CVD scores $<3$ compared to those with CVD scores $\geq 3$ (Table 1).

Rate of Surgery: The rates of surgery (PDA ligation) in the groups with CVD scores $\geq 3(16.5 \%)$, whereas none of the patients with CVD scores $<3$ received surgery (Table 1 ).

\section{Clinical outcomes according to CVD scores $\geq 3$}

IIncidence of PDA Closure: According to Figure 1 and Table 2, in patients with initial CVD scores $\geq 3$, the rates of PDA closure in the conservational management (B1) group were only $6.7 \%$, being significantly different from $69.8 \%$ in the ibuprofen intervention groups $(P<0.0001)$.

PDA closure and clinical outcomes are associated with intervention timing of ibuprofen: The incidence rate of PDA closure in the early ibuprofen treatment group B2 was significantly different from that in the late ibuprofen treatment group B3 $(16.7 \%$ vs $70.9 \%, P<0.0001)$, but the differences of incidence rates of BPD $(25 \%$ vs $19.9 \%, P=0.442)$, IVH (33.3\% vs $23.7 \%, P=0.318)$, ROP $(41.7 \%$ vs $19.4 \%, P=0.056)$ and NEC (0 vs $1.3 \%)$ between the two groups did not reach statistical significance (Table 2). 


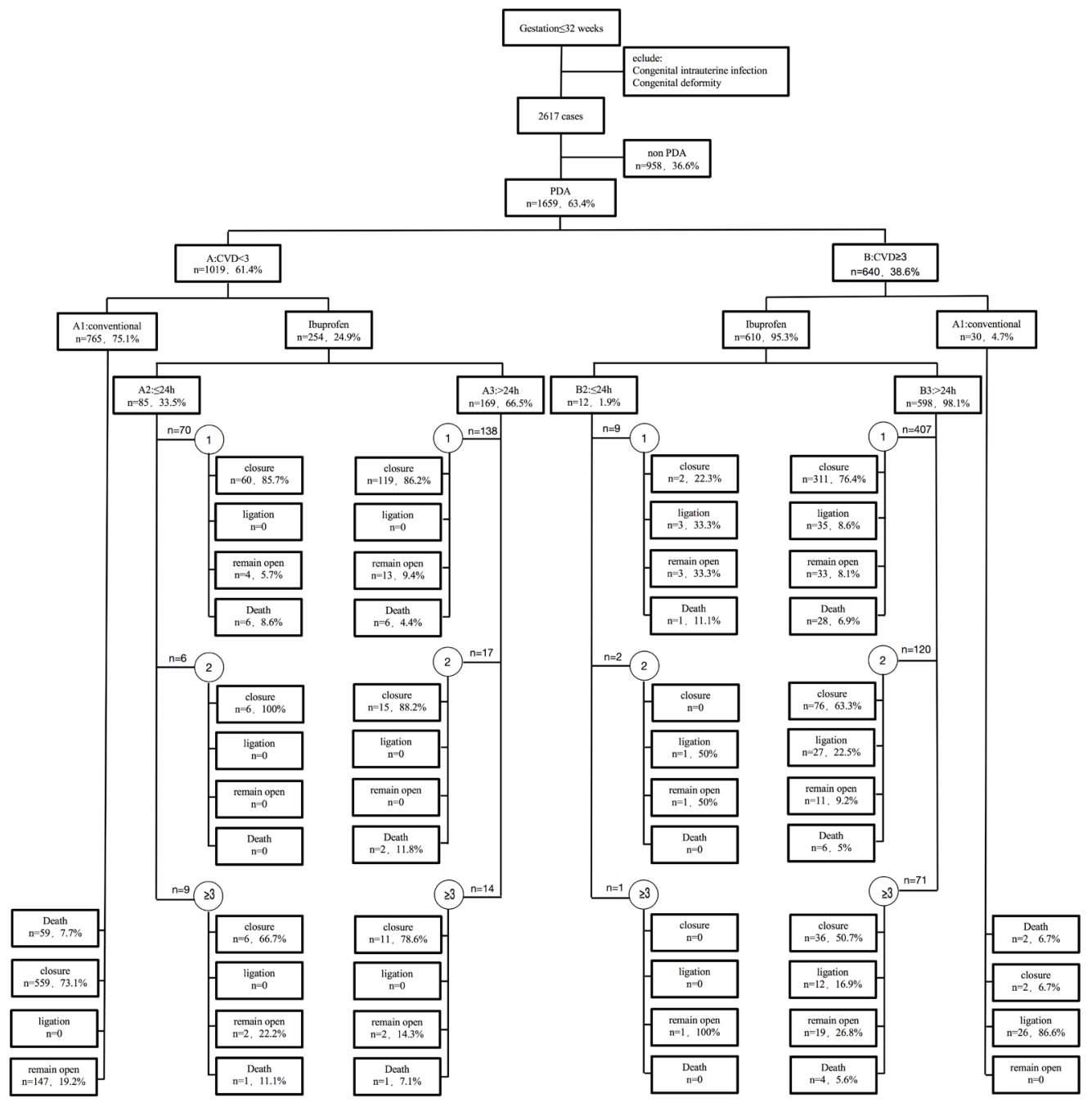

Figure 1. Study flow diagram

Rate of Surgery: According to Table 2, the rates of surgery in the B2 and B3 groups were $12.6 \%$, which were significantly different from $86.6 \%$ in the conservational management group B1 $(P<$ 0.000 1). However, the surgery rate in $B 2$ was numerically, but not statistically, higher than that in $\mathrm{B} 3(33.3 \%$ vs $12.2 \%, P=0.053)$.

Clinical outcomes according to the course of treatment: The rates of PDA closure in B3 decreased linearly with increasing course of ibuprofen ( $1^{\text {st }}$ course vs $2^{\text {nd }}$ course vs $3^{\text {rd }}$ course: $76.4 \%$ vs $63.3 \%$ vs $\left.50.7 \%, \chi^{2}=9.632, P<0.0001\right)$. In sharp contrast, the rates of NRDS ( $1^{\text {st }}$ course vs $2^{\text {nd }}$ course vs $3^{\text {rd }}$ course: $79.9 \%$ vs $89.3 \%$ vs $\left.90.1 \%, \chi^{2}=6.920, P=0.012\right)$, ROP $\left(1^{\text {st }}\right.$ course vs $2^{\text {nd }}$ course $3^{\text {rd }}$ course: $16.5 \%$ vs $23.8 \%$ vs $\left.29.6 \%, \chi^{2}=6.978, P=0.016\right)$, and BPD $\left(1^{\text {st }}\right.$ course vs $2^{\text {nd }}$ course vs $3^{\text {rd }}$ course: $15.5 \%$ vs $26.7 \%$ vs $33.8 \%$, $\chi^{2}=18.390, P<0.0001$ ) increased linearly with increasing course of ibuprofen treatment. The rates of IVH ( $1^{\text {st }}$ course vs $2^{\text {nd }}$ course vs $3^{\text {rd }}$ course: $20.4 \%$ vs $32.4 \%$ vs $\left.23.8 \%, \chi^{2}=7.071, P=0.015\right)$ and surgical treatment ( $1^{\text {st }}$ course vs $2^{\text {nd }}$ course vs $3^{\text {rd }}$ course: $8.6 \%$ vs $27 \%$ vs $16.9 \%, \chi^{2}=10.721, P<0.0001$ ) were independent of the course of ibuprofen (Table 3 ).

\section{Discussion}

It has been reported that around $25 \%$ to $59 \%$ of VPIs or very low birth weight infants have PDA that fail to spontaneously close[1, $5,22]$. In this large cohort study, we found that the incidence of PDA in VPIs was $63.4 \%$. Our study differs from published studies in that we applied the CVD scoring to stratify the possibility of spontaneous PDA closure. Specifically, we found that VPIs with CVD scores consistently $<3$ had higher rates of spontaneous PDA closure which requires conservative management instead of ibuprofen interventions. However, VPIs with CVD scores 
Table 1. Clinical outcomes according to CVD scores

\begin{tabular}{|c|c|c|c|c|}
\hline \multirow{3}{*}{ Variables } & \multicolumn{2}{|c|}{ PDA } & \multirow{3}{*}{$\chi^{2} / \mathrm{t}$} & \multirow{3}{*}{$P$ value } \\
\hline & CVD $<3$ & $C V D \geq 3$ & & \\
\hline & $n=1019$ & $n=640$ & & \\
\hline Surgery(PDA ligation )(n/\%) & 0 & 16.5 & - & - \\
\hline PDA closure (n/\%) & $75.0(772)$ & $66.5(426)$ & 16.201 & $<0.0001$ \\
\hline $1^{\text {st }}$ course of ibuprofen $(n / \%)$ & 86.1(179) & $75.2(313)$ & 9.439 & 0.002 \\
\hline $2^{\text {nd }}$ course of ibuprofen $(n / \%)$ & $91.3(21)$ & $62.3(76)$ & 1.136 & 0.287 \\
\hline $3^{\text {rd }}$ course of ibuprofen $(n / \%)$ & $73.9(17)$ & $50.0(36)$ & 4.041 & 0.044 \\
\hline $\operatorname{RDS}(n / \%)$ & $66.8(679)$ & $83.5(533)$ & 56.219 & $<0.0001$ \\
\hline $\operatorname{ROP}(\mathrm{n} / \%)$ & $7.7(77)$ & $20.7(131)$ & 59.941 & $<0.0001$ \\
\hline IVH (n/\%) & $17.4(179)$ & $25(159)$ & 12.881 & $<0.0001$ \\
\hline $\operatorname{NEC}(n / \%)$ & $1.1(10)$ & $1.2(8)$ & 0.264 & 0.607 \\
\hline BPD (n/\%) & $8.9(90)$ & $22.4(143)$ & 59.539 & $<0.0001$ \\
\hline Death $(n / \%)$ & $8.8(75)$ & $6.8(41)$ & 0.538 & 0.463 \\
\hline PDA closure time (d) & $10.8 \pm 0.8$ & $14.5 \pm 1.4$ & 7.015 & $<0.0001$ \\
\hline PDA diameter (mm) & $2.2 \pm 0.5$ & $2.4 \pm 0.6$ & 6.280 & $<0.0001$ \\
\hline Ibuprofen (n/\%) & $24.9(254)$ & $95.3(610)$ & 779.560 & $<0.0001$ \\
\hline Ibuprofen after $24 \mathrm{~h}(\mathrm{n} / \%)$ & $66.5(169)$ & $98.1(598)$ & 178.508 & $<0.0001$ \\
\hline
\end{tabular}

BPD, pulmonary dysplasia; ROP, retinopathy of prematurity; IVH. intraventricular hemorrhage; NEC, Necrotizing enterocolitis.

Table 2. Clinical outcomes according to CVD scores $\geq 3$

\begin{tabular}{|c|c|c|c|c|}
\hline \multirow{2}{*}{ Variables } & \multicolumn{4}{|c|}{ CVD $\geq 3$} \\
\hline & B1 vs B2 & B1 vs B3 & B2 vs B3 & $\mathrm{B} 2+\mathrm{B} 3$ vs $\mathrm{B} 1$ \\
\hline \multirow{2}{*}{ PDA ligation (\%) } & $<0.0001$ & $<0.0001$ & 0.053 & $<0.0001$ \\
\hline & (96.7 vs 33.3) & (96.7 vs 12.2) & (33.3 vs 12.2) & (12.6 vs 86.6) \\
\hline \multirow{2}{*}{ PDA closure (\%) } & 0.192 & $<0.0001$ & $<0.001$ & $<0.0001$ \\
\hline & (6.7 vs 16.7 ) & (6.7 vs 70.9$)$ & (16.7 vs 70.9 ) & (69.8 vs 6.7) \\
\hline \multirow{2}{*}{ ROP (\%) } & 0.730 & 0.048 & 0.056 & 0.057 \\
\hline & (34.5 vs 41.7) & (34.5 vs 19.4) & (41.7 vs 19.4) & (19.9 vs 34.5$)$ \\
\hline \multirow{2}{*}{ IVH (\%) } & 0.729 & 0.010 & 0.318 & 0.11 \\
\hline & (44.8 vs 33.3 ) & (44.8 vs 23.7 ) & (33.3 vs 23.7 ) & (23.9 vs 44.8$)$ \\
\hline \multirow{2}{*}{ NEC (\%) } & - & - & - & - \\
\hline & (0 vs 0$)$ & $(0$ vs 0$)$ & (0 vs 1.3) & (1.3 vs 0$)$ \\
\hline \multirow{2}{*}{ BPD (\%) } & 0.005 & $<0.0001$ & 0.442 & $<0.0001$ \\
\hline & (72.4 vs 25) & (72.4 vs 19.9) & (25 vs 19.9$)$ & (20.0 vs 72.4$)$ \\
\hline \multirow{2}{*}{ Death (\%) } & 1.000 & 0.947 & 0.551 & 1.000 \\
\hline & (6.7 vs 8.3$)$ & (6.7 vs 6.4$)$ & (8.3 vs 6.4$)$ & (6.4 vs 6.7 ) \\
\hline \multirow{2}{*}{$\begin{array}{c}\text { PDA } \\
\text { closure time (d) }\end{array}$} & 0.307 & 0.028 & 0.554 & 0.026 \\
\hline & $(19.2 \pm 6.2$ vs $11.4 \pm 0.2)$ & $(19.2 \pm 6.2$ vs $14.3 \pm 1.1)$ & $(11.4 \pm 0.2$ vs $14.3 \pm 1.1)$ & $(19.2 \pm 6.2$ vs $14.3 \pm 1.1)$ \\
\hline \multirow{2}{*}{$\begin{array}{c}\text { PDA } \\
\text { diameter }(\mathrm{mm})\end{array}$} & 0.622 & 0.612 & 0.463 & 0.625 \\
\hline & $(2.4 \pm 0.5$ vs $2.5 \pm 0.5)$ & $(2.4 \pm 0.5$ vs $2.3 \pm 0.7)$ & $(2.5 \pm 0.5$ vs $2.4 \pm 0.7))$ & $(2.4 \pm 0.5$ vs $2.3 \pm 0.7)$ \\
\hline
\end{tabular}

$B P D$, pulmonary dysplasia; ROP, retinopathy of prematurity; IVH, intraventricular hemorrhage; NEC, Necrotizing enterocolitis.

B1, non-ibuprofen intervention; B2, ibuprofen intervention was conducted within $24 h$; B3, ibuprofen intervention was conducted after 24h; B2+B3, ibuprofen intervention 
Table 3. Clinical outcomes according to ibuprofen course and intervention timing

\begin{tabular}{|c|c|c|c|c|c|c|c|c|}
\hline \multirow{3}{*}{$\begin{array}{c}\text { CVD score } \\
\text { Time } \\
\text { Course }\end{array}$} & \multicolumn{8}{|c|}{$\geq 3$} \\
\hline & \multicolumn{4}{|c|}{ B2 $(<24 h)$} & \multicolumn{4}{|c|}{ B3 ( $\geq 24 h)$} \\
\hline & 1 & 2 & $\geq 3$ & $\mathrm{P}$ & 1 & 2 & $\geq 3$ & $\mathrm{P}$ \\
\hline Antenatal & 66.7 & 50 & 0 & - & 37.0 & 39.7 & 39.4 & 0.828 \\
\hline \multicolumn{9}{|l|}{ Corticosteroid (\%) } \\
\hline NRDS (\%) & 66.7 & 100 & 100 & 0.513 & 79.9 & 89.3 & 90.1 & 0.012 \\
\hline Pneumonia (\%) & 77.8 & 100 & 0 & - & 29.5 & 46.7 & 31.0 & 0.002 \\
\hline Closure (\%) & 22.2 & 0 & 0 & - & 76.4 & 63.3 & 50.7 & $<0.0001$ \\
\hline Ligation (\%) & 33.3 & 50.0 & 0 & - & 8.6 & 27 & 16.9 & $<0.0001$ \\
\hline ROP (\%) & 44.4 & 50 & 0 & - & 16.5 & 23.8 & 29.6 & 0.016 \\
\hline IVH (\%) & 11.1 & 10.0 & 1 & 0.018 & 20.4 & 32.4 & 23.8 & 0.015 \\
\hline NEC (\%) & 0 & 0 & 0 & - & 1.7 & 0.8 & 0 & - \\
\hline BPD (\%) & 33.3 & - & - & - & 15.5 & 26.7 & 33.8 & $<0.0001$ \\
\hline Death (\%) & 11.1 & - & - & - & 6.9 & 5.0 & 5.6 & 0.743 \\
\hline
\end{tabular}

RDS, respiratory distress syndrome; BPD, pulmonary dysplasia; ROP, retinopathy of prematurity; IVH, intraventricular hemorrhage; NEC, Necrotizing enterocolitis.

B2, ibuprofen intervention was conducted within $24 \mathrm{~h}$; B3, ibuprofen intervention was conducted after $24 \mathrm{~h}$

consistently $\geq 3$ were more likely to develop significant ductal shunt, and intervention with ibuprofen may be indicated. These results highlight the potential importance of applying the CVD score to predict PDA closure rate in VPIs, particularly when hemodynamic assessments are unavailable.

Administration of ibuprofen to neonates yielded a PDA closure rate of $74.3 \%$, which is consistent with the results reported by published data[23-24]. Interestingly, in preterm infants who carry many pre-natal and post-natal risks[25], the closure rate after administration of ibuprofen may be high. This is consistent with the consensus that orally administered ibuprofen can effectively promote the closure of PDA, reduce significant ductal shunt, and prevent unnecessary surgical PDA ligation as evidenced by the results from the studies by Ohlsson et al.[26] and Shah et al[27]. However, as ibuprofen might result in digestive tract or transient kidney injury, we sought to determine how ibuprofen can be used in multiple courses without increasing side effects.

PDA closure following ibuprofen decreased with incremental courses of treatment. However, studies that utilized the same ibuprofen treatment course showed that after the third course, some neonates did not achieve PDA closure especially those with sustained CVD score $\geq 3$, and the risks of IVH, ROP, and BPD were significantly increased[28]. These results support our conclusion that PDA persistence contributes to the development of IVH, ROP, and BPD. And in these same infants, the longer they suffered PDA (CVD scores $\geq 3$ ), the more preterm complications they would have. We did not observe any complications related to transient kidney injury, which could presumably arise from oral administration of ibuprofen.

Moreover, we found that administration of ibuprofen within $24 \mathrm{~h}$ after conservational management showed similar incidence rates of BPD, ROP, NEC and IVH to those in patients treated with ibuprofen after $24 \mathrm{~h}$ of conservational management. These results indicated little benefit of early PDA treatment at the onset of mild clinical signs. This is inconsistent with the findings of Van Overmeire et al[29] who showed that prophylactic ibuprofen treatment improved the PDA closure rate without significantly affecting the risk of IVH. Similarly, our results are also inconsistent with a meta-analysis showing that prophylactic ibuprofen can promote PDA closure and reduce complications in VPIs[28]. Therefore, for VPIs with PDA that self-closes, indiscriminate administration of ibuprofen is not recommended. Based on our findings, we proposed a strategy for effectively identifing neonates who would benefit from ibuprofen administration, which is of clinical significance.

In our research, we included the CVD score as an index for hemodynamic PDA scoring. It should be noted that preterms who originally are classified with CVD scores $<3$ can progress to CVD scores $\geq 3$ after ibuprofen administration. These infants likely require ventilation and extensive use of furosemide, as their PDA may self-close. Ibuprofen is suggested for PDA closure only when the PDA reopens or has developed into the stage with the CVD score $\geq 3$. llene et al suggest that there is little benefit of early PDA treatment at the onset of mild clinical signs compared to delayed PDA treatment until there is an onset of clear hemodynamic signs[8]. For CVD scores that are continuously $\geq 3$, in the absence 
of ibuprofen intervention the rate of surgical treatment to achieve PDA closure was as high as $86.6 \%$, and the incidences of ROP, IVH, BPD, NRDS, and pneumonia also increased accordingly. The presence of severe clinical symptoms may hinder the ability of medicinal intervention to close PDA and result in an increased risk of complications and rate of surgical ligation. In VPIs whose CVD scores were $\geq 3$, the closure rate with late PDA treatment (B3 group) was greater than that in those with early PDA treatment (B2 group). Even after 2 courses of treatment, PDA could still close in some VPIs. Therefore, ibuprofen can be applied under the guidance of the CVD score for more than 3 courses of treatment.

However, we found that the incidence of clinical outcomes, including BPD and ROP, were higher in neonates who received ibuprofen compared to those who did not. These results were confirmed by a recent meta-analysis that demonstrated an increased risk of BPD with ibuprofen compared to indomethacin treatment[30]. In our point of view, increased BPD in neonates receiving ibuprofen may be related to lower rates of receiving antenatal corticosteroids[25] and longer duration in the severe defect state. Therefore, for VPIs with an antenatal corticosteroid deficiency, a post-natal history of hypoxia, and a sustained CVD score $\geq 3$, ibuprofen must be administered in a timely manner, not prophylactic, to promote PDA closure, improve respiration, and decrease risk of complications.

Survival of premature infants has increased over the past decades, attributing to an increase in PDA prevalence at birth[31]. China is one of the top five countries with relative high rate of preterm births accounting for approximately $12 \%$ of the global preterm births in 2014[31]. When navigating to the cold areas within China, the preterm birth rates can soar up to $18.49 \%$. Moreover, Białkowski and his colleagues reported that patients with PDA living at high altitude are more prone to exhibit larger ductal diameter and higher pulmonary artery pressure[32]. As it happens, the majority of cold areas in China are also at high altitudes. The above evidence suggests that cold areas and high altitude are independent risk factors of decreased spontaneous PDA closure. Therefore, it is imperative to use objective index for hemodynamic PDA evaluation. The CVD scoring may be a significant predictor of PDA closure rate in VPIs, particularly in cold areas with high altitude.

This study has limitations in that it is a retrospective and included a relatively short median follow-up duration. In order to elucidate the safety and efficacy of ibuprofen in VPIs with PDA, welldesigned RCTs and multi-center relevant to severe cold area with high altitude are warranted. Notably, the strengths of the present study include a large-scale sample size and complete clinical data from an established database, which may have minimized the selection bias and information bias. Each of the criteria for CVD scoring was objectively and routinely assessed in infant with PDA in our NICU. Therefore, the changes of the scores in each infant with time or differences of the scores between the infants can reflex meaningful changes in hemodynamics.

\section{Conclusions}

In conclusion, intervention with ibuprofen can increase the rate of PDA closure in selected preterm neonates, and the CVD scoring may be a significant predictor of PDA closure rate in VPIs, particularly when hemodynamic assessments are unavailable. Clinicians could consider using the CVD scoring as a tool to help determine the timing, frequency, and duration of ibuprofen treatment in VPI's as a criterion for referral for surgery when ibuprofen is ineffective.

\section{Acknowledgement}

Zhi-Chun Feng and Jia Chen conceptualized and designed the study, supervised data collection, and contributed to the analysis, interpretation, and first and final draft of the manuscript. Jia Chen and Jinghua Luo designed the collection instruments. Jinghua Luo, Xue Du, Shan Zhang, Zizhen Wang and Liu He collected the data. Jia Chen and Hao Liu performed the statistical analysis. Al authors approved the final manuscript as submitted and agreed to be accountable for all aspects of the work. We appreciate Mr. Chen Daiqiu from the English Dept. of Southern Medical University for the language comments on this manuscript.

\section{Ethical approval}

This study was approved by the Medical Ethics Committee of the Clinical Medical College in PLA Army Hospital, and informed consent in writing was provided by the legal guardians of the VPIs (No: 2017.50).

\section{Registration}

Chinese Clinical Trail Registry, ChiCTR2000032121.

\section{Patient consent for publication}

We obtained written informed consent from infant's parents.

\section{Conflict of interests}

All authors showed no conflicts of interest. No financial or nonfinancial benefits have been received or will be received from any party related directly or indirectly to the subject of this article. 


\section{References}

[1] Clyman R I, Couto J, Murphy G M. Patent ductus arteriosus: are current neonatal treatment options better or worse than no treatment at all? Seminars in Perinatology, 2012; 36(2): 123-129.

[2] Hamrick S E G, Hansmann G. Patent ductus arteriosus of the preterm infant. Pediatrics, 2020; 125(5): 1020-1030.

[3] Gournay V. The ductus arteriosus: Physiology, regulation, and functional and congenital anomalies. Archives of Cardiovascular Disease, 2011; 104(11): 578-585.

[4] Brown E R. Increased risk of bronchopulmonary dysplasia in infants with patent ductus arterosis. J Pediatr, 1979; 95(5 Pt 2): 865-866.

[5] Hagadorn J I, Brownell E A, Trzaski J M, et al. Trends and variation in management and outcomes of very low birth weight infants with patent ductus arteriosus. Pediatric Research, 2016; 80(6): 785-792.

[6] Lipman B, Serwer G A, Brazy J E. Abnormal cerebral hemodynamics in preterm infants with patent ductus arteriosus, 1982; 69(6): 778-781.

[7] Sweet D G, Carnielli V, Greisen G, et al. European consensus guidelines on the management of respiratory distress syndrome-2016 Update. Neonatology, 2017; 111(2): 107-125.

[8] Sosenko I R S, Fajardo M F, Claure N, et al. Timing of patent ductus arteriosus treatment and respiratory outcome in premature infants: a double-blind randomized controlled trial. Journal of Pediatrics, 2012; 160(6): 929-935.

[9] Letshwiti J B, Semberova J, Pichova K, et al. A conservative treatment of patent ductus arteriosus in very low birth weight infants. Early Human Development, 2017; 104: 45-49.

[10] Mohamed M A, El-Dib M, Alqahtani S, et al. Patent ductus arteriosus in premature infants: to treat or not to treat? Journal of Perinatology, 2017; 37(6): 652-657.

[11] Perez K M, Laughon M M. What is new for patent ductus arteriosus management in premature infants in 2015? Current Opinion in Pediatrics, 2015; 27(2): 158-164.

[12] Yeh T F, Luken J A, Thalji A, et al. Intravenous indomethacin therapy in premature infants with persistent ductus arteriosus-a double-blind controlled study. J Pediatr, 1981; 98(1): 137-145.

[13] Yeh T F, Thalji A, Luken L, et al. Improved lung compliance following indomethacin therapy in premature infants with persistent ductus arteriosus. Chest, 1981; 80(6): 698-700.

[14] Yeh T F, Raval D, Luken J, et al. Clinical evaluation of premature infants with patent ductus arteriosus: a scoring system with echocardiogram, acid-base, and blood gas correlations. Critical Care Medicine. 1981; 9(9): 655-657.

[15] Afiune J Y, Singer J M, Leone C R. Echocardiographic post-neonatal progress of preterm neonates with patent ductus arteriosus. Jornal De Pediatria, 2005; 81(6): 454-460.

[16] Baraldi E, Filippone M. Chronic lung disease after premature birth. The New England journal of medicine, 2007; 357(19): 1946.

[17] Mccrea H J, Ment L R. The diagnosis, management, and postnatal prevention of intraventricular hemorrhage in the preterm neonate. Clinics in Perinatology, 2008; 35(4): 777-792.

[18] Thompson A M, Bizzarro M J. Necrotizing enterocolitis in newborns: pathogenesis, prevention and management. Drugs, 2008; 68(9): 1227-1238. [19] Haines L, Fielder A R, Baker $\mathrm{H}$, et al. UK population based study of severe retinopathy of prematurity: screening, treatment, and outcome. Archives of Disease in Childhood Fetal and Neonatal Edition, 2005; 90(3): F240-F244.

[20] Sweet D G, Carnielli, V P, Greisen G, et al. European consensus guidelines on the management of neonatal respiratory distress syndrome in preterm infants-2010 update. Neonatology, 2010; 97(4): 402-417.

[21] Erdeve O, Yurttutan S, Altug N, et al. Oral versus intravenous ibuprofen for patent ductus arteriosus closure: a randomised controlled trial in extremely low birthweight infants. Arch dis child fetal neonatal ed, 2012; 97(4): F279-F283.

[22] Koch J, Hensley G, Rosenfeld C R. Prevalence of spontaneous closure of the ductus arteriosus in neonates at a birth weight of 1000 grams or less. Pediatrics, 2006; 117(4): 1113-1121.

[23] Adrouche-Amrani L, Green R S, Gluck K M, et al. Failure of a repeat course of cyclooxygenase inhibitor to close a PDA is a risk factor for developing chronic lung disease in ELBW infants. BMC Pediatr, 2012; 27(12): 10.

[24] Gournay V, Roze J C, Kuster A, et al. Prophylactic ibuprofen versus placebo in very premature infants: a randomised, double-blind, placebocontrolled trial. Lancet, 2004; 364(9449): 1939-1944.

[25] Overmeire B V, Allegaert K, Casaer A, et al. Prophylactic ibuprofen in premature infants: A multicentre, randomised, double-blind, placebocontrolled trial. Journal of Pediatrics, 2004; 364(9449): 1945-1949.

[26] Clyman R I. Recommendations for the postnatal use of indomethacin: An analysis of four separate treatment strategies. Journal of Pediatrics, 1996; 128(5 Pt 1): 601-607.

[27] Ohlsson A, Shah S. Ibuprofen for the prevention of patent ductus arteriosus in preterm and/or low birth weight infants. The Cochrane database of systematic reviews, 2019; 6: CD004213.

[28] Mitra S, Florez I D, Tamayo M E, et al. Association of placebo, indomethacin, ibuprofen, and acetaminophen with closure of hemodynamically significant patent ductus arteriosus in preterm infants. Jama the Journal of the American Medical Association, 2018; 319(12):1221.

[29] Overmeire B V, Smets K, Lecoutere D. A comparison of ibuprofen and indomethacin for closure of patent ductus arteriosus. Acc Current Journal Review, 2000; 34310(1):674-681.

[30] Garingo A, Tesoriero L, Cayabyab R, et al. Constitutive IL-10 expression by lung inflammatory cells and risk for bronchopulmonary dysplasia. Pediatric research, 2007; 61(2):197-202.

[31] Van der Linde D, Konings E E, Slager M A, et al. Birth prevalence of congenital heart disease worldwide: a systematic review and meta-analysis. Journal of the American College of Cardiology, 2011; 58(21):2241-2247.

[32] Białkowski J, Głowacki J, Zabal C, et al. Patent ductus arteriosus at low and high altitudes: anatomical and haemodynamic features and their implications for transcatheter closure. Kardiologia polska, 2011; 69(5):431-436. 